\title{
Analyzing the Physico-Chemical Parameters of Detergents and Detergent Mixtures
}

\author{
Britta Tschapek, Sander H. J. Smits, Lutz Schmitt* \\ Institute of Biochemistry, Heinrich Heine University Duesseldorf, \\ Duesseldorf, Germany \\ Email: [Lutz.Schmitt@hhu.de
}

Received 2 June 2015; accepted 5 July 2015; published 9 July 2015

Copyright (C) 2015 by authors and Scientific Research Publishing Inc.

This work is licensed under the Creative Commons Attribution International License (CC BY). http://creativecommons.org/licenses/by/4.0/

(c) (i) Open Access

\section{Abstract}

The most important physico-chemical characteristics describing detergent micelles are the CMC value and the aggregation number. These parameters depend critically on external conditions such as $\mathbf{p H}$, ionic strength, buffer composition or temperature. However, the influence of these conditions on CMC or aggregation number is a priori not to predict, but the most important parameter. This holds especially for the aggregation number. Here, we present a simple, reliable and fast method for the analysis of detergent containing buffer systems. It enables the determination of CMC values and aggregation numbers of detergents as well as detergent mixtures in a 96well plate standard device relying on steady state fluorescence and fluorescence quenching. To prove the general applicability of our approach, we analyzed DDM as a prime example of a detergent and mixtures of DDM with six other detergents at different ratios to demonstrate the potential of our method.

\section{Keywords}

Detergent, CMC Determination, Fluorescence, Mixed Micelles, Aggregation Number

\section{Introduction}

Detergents are widespread tools in many active areas of research including material and surface sciences as well as life sciences. In the latter, detergents are important prerequisites for an in vitro analysis of integral membrane proteins (IMP). Initially, IMPs are solubilized from cellular membranes and further purified until biochemical, biophysical or even structural studies can be performed. Each of these steps requires a stable and homogenous IMP in detergent solution. Often not a single detergent is used for all intermediate steps, in some cases the de-

\footnotetext{
${ }^{*}$ Corresponding author.
}

How to cite this paper: Tschapek, B., Smits, S.H.J. and Schmitt, L. (2015) Analyzing the Physico-Chemical Parameters of Detergents and Detergent Mixtures. Advances in Chemical Engineering and Science, 5, 328-337. 
tergent is exchanged during purification or even detergent mixtures are used to keep the IMP stable in solution. One underestimated parameter pitfall lies within the physical behavior of detergents in buffers of different ionic strengths [1] [2]. For example, the critical micellar concentration (CMC) differs for DDM in a buffer with no salt compared to for example a $250 \mathrm{mM}$ sodium chloride solution. Although and in comparison to other detergents, the change of the CMC for DDM from 0.12 to $0.098 \mathrm{mM}$ is rather small [1], the effect of salt on a solution containing a single or multiple detergent(s) is complicated and cannot be estimated. Another important parameter of a detergent micelle is the aggregation number. Changes in the physico-chemical condition of the buffer (temperature, $\mathrm{pH}$, ionic strength or additives) might change the aggregation number of the micelle in an unpredictable fashion.

The choice of the "right" detergent can be crucial, as shown for the $\mathrm{Ca}^{2+}$-ATPase from Sarcoplasmic Reticulum. For this membrane protein, more than 30 detergents have been tested for preserving the activity of this IMP. The analysis focused on chain length, influence of double bonds and character of the head group. A reduced activity was observed for shorter chain length ( $<10 \mathrm{C}$-Atoms) and bulky head groups just to mention two examples of this study [3]. Also for the multidrug transporter LmrA from Lactococcus lactis the choice of detergent has been shown to be important. Here, LmrA after solubilisation is active in FC16 whereas after reconstitution it is not. For successful reconstitution, the detergent DDM had to be used for regaining LmrA specific activity [4].

Optimal detergent (or detergent mixture) conditions for IMPs cannot be predicted and an analysis is required for every single IMP or for each individual purification step. Due to the hydrophilic and hydrophobic parts of membrane proteins the exact size of the detergent micelle can play a crucial role for any experiment designed after the IMP has been purified. Therefore, the most detailed knowledge of the physico-chemical parameters (CMC or aggregation number) of the detergent used in these studies is of great help when screening for suitable conditions.

An example for such a screening procedure was described for the crystallization of the $\mathrm{Na}^{+} / \mathrm{H}^{+}$antiporter NhaA from E. coli [5]. Here, 14 different detergents were screened in the crystallization process yielding different crystal forms resulting in varying quality of X-ray diffraction. Finally, a mixture of $\alpha$-dodecylmaltoside (DDM) and $\beta$-octyl-glucoside (OG) yielded the best diffracting crystals. The author's assumption is that the addition of $\beta$-OG to NhaA purified in $\alpha$-DDM reduced the micelle size thereby allowing the formation of stable crystal contacts [5]. This phenomenon has been suggested as a general strategy for the crystallization of membrane proteins (for other examples see also [6] [7]).

Here, we present a simple "tool-box" combining different, based on already published procedures [1] [8]-[10] to determine the CMC values as well as and most importantly the aggregation numbers of detergents or detergent mixtures. As a representative detergent, we choose DDM as this is the most widely used detergent in membrane protein purification, analysis and crystallization [11].

Several studies have shown that pyrene quenching is suitable to determine aggregation numbers of detergents [8] [9] [12]-[14]. However, in most biochemical laboratories this is not commonly used, because the above referenced methods are time consuming and require high sophisticated fluorescence equipment. With our methodusing 96well standard microtiter plates — it is possible to analyze most properties of the detergent micelles in any buffer system. This can provide valuable information for example when screening for reduced micelle size, or to visualize the amount of free detergent within only a couple of hours.

\section{Materials and Methods}

Triton X-100, LDAO, and Pyrene were purchased from Sigma Chemicals. All other detergents used in this study were received from Affymetrix.

\subsection{Fluorescence Measurements}

\subsubsection{Determination of the CMC Value}

CMCs were determined according to the set-up described earlier [1]. In brief, Hoechst33342 is highly fluorescence in hydrophobic environment whereas it hardly emits fluorescence in aqueous solution. Therefore, Hoechst33342 fluorescence was monitored at increasing detergent concentrations. When fluorescence starts to increase (first inflection point), micelles start to form and an interpolation to the X-axis leads to the CMC value. For further details see Equation (1). 


\subsubsection{Determination of the Partitioning Coefficient}

The partitioning coefficient of Hoechst33342 $\left(P_{m(\text { Hoechst } 33342)}\right)$ between the aqueous $(w)$ and micellar phase $(m)$ is given by Equation (1):

$$
P_{m(\text { Hoechst } 33342)}=\frac{[\text { Hoechst } 33342]_{m}}{[\text { Hoechst } 33342]_{w}}
$$

As described earlier [8], it is possible to calculate the partitioning coefficient by measuring the fluorescence intensity of the dye at different $\alpha_{m}$ values. $\alpha_{m}$ corresponds to the ratio of micelle volume to total volume of the aqueous solution. If $F_{m}>>F_{w}$, where $\mathrm{F}$ is the fluorescence of Hoechst33342, one obtains Equation (2):

$$
\frac{F}{F_{\infty}}=\frac{a_{m}[\text { Hoechst33342 }]_{m}}{a_{m}[\text { Hoechst33342 }]_{m}+\left(1-a_{m}\right) \frac{[\text { Hoechst33342 }]_{m}}{P m}}
$$

$F_{\infty}$ corresponds to the fluorescence when all Hoechst33342 molecules are located in micelles. A plot of the inverse fluorescence versus inverse $\alpha_{m}$ yields a linear function, which provides the partitioning coefficient as the intercept divided by the slope (Equation (3)).

$$
\frac{1}{F}=\frac{1}{F_{\infty}}-\frac{1}{P_{m} F_{\infty}}+\left(\frac{1}{P_{m} F_{\infty}} \cdot \frac{1}{a_{m}}\right)
$$

\subsubsection{Steady State Fluorescence Quenching Studies}

Detergent concentrations used in the assay were five folds above the CMC, determined with the method mentioned above, except for detergents with a very high $\mathrm{CMC}(\geq 5 \mathrm{mM})$. In these cases, the detergent concentration was adjusted to $2 \mathrm{mM}$ above CMC. For example, the aggregation number of SDS was measured at $10 \mathrm{mM}$ $(\mathrm{CMC}=8 \mathrm{mM})$. Pyrene concentration was adjusted to at least 10 fold below the concentration of micelles present as calculated from literature values for the aggregation number to avoid excimer formation. The quencher concentration varied from 0 to $20 \mu \mathrm{M}$.

Steady-state fluorescence quenching experiments were carried out either using a Fluorolog-3 (Horiba Jobin Yvon GmbH, Germany, Unterhaching) or a 96well plate reader (Fluorostar, BMG labtech, Offenburg, Germany). Excitation wavelength was set to $335 \mathrm{~nm}$ (filter: $330 \pm 10 \mathrm{~nm}$ ), emission was monitored from 365 to 450 $\mathrm{nm}$ or with a filter of $372 \pm 10 \mathrm{~nm}$. Fluorescence data were corrected for background fluorescence and analyzed according to Equation (4):

$$
\ln \frac{I}{I_{0}}=-\frac{N[Q]}{\left(C_{t}-c m c\right)}
$$

Here, $I$ and $I_{o}$ are the intensities of the first vibronic peak of the fluorescence emission spectra of pyrene in the presence $(I)$ and absence $\left(I_{0}\right)$ of varying quencher concentrations. $C_{1}$ is the total detergent concentration, $\mathrm{N}$ the aggregation number and CMC is the critical micellar concentration [12].

\subsubsection{Theoretical Treatment of Mixed Detergent Systems: Ideal Mixing}

To analyze whether it is necessary to measure the CMC of the mixed detergent system or if one can rely on theoretical data, we compared our data with the ideal mixing model [15]. This model relates the CMC of an ideal detergent mixture $\left(\mathrm{CMC}^{*}\right)$ with the experimental $\mathrm{CMC}$ of the pure components using the following equations:

$$
c_{1}=x_{1} \mathrm{CMC}_{1}=a_{1} \mathrm{CMC}^{*}
$$

and

$$
c_{2}=x_{2} \mathrm{CMC}_{2}=\left(1-a_{1}\right) \mathrm{CMC}^{*}
$$

Equation (7) can be obtained by combining Equations (5) and (6):

$$
\frac{1}{\mathrm{CMC}}=\frac{\alpha_{1}}{\mathrm{CMC}_{1}}+\frac{1-\alpha_{1}}{\mathrm{CMC}_{2}}
$$


where $\alpha_{\mathrm{i}}$ is the amount of detergent $i$ in aqueous solution and $x_{i}$ is the amount of detergent $i$ in the micelles in aqueous solute or in the mixed micelle. $\mathrm{CMC}_{1}$ and $\mathrm{CMC}_{2}, c_{1}$ and $c_{2}$ are the CMCs and the overall concentrations of detergent 1 and 2, respectively. If both detergents do not obey ideal mixing, one has to add a correction factor, the micellar activity coefficient $\mathrm{f}$ :

$$
\frac{1}{\mathrm{CMC}}=\frac{\alpha_{1}}{f_{1} \mathrm{CMC}_{1}}+\frac{\left(1-\alpha_{1}\right)}{f_{2} \mathrm{CMC}_{2}}
$$

For a more detailed description of this theory, see Sarmoria et al. [16] for further details.

\section{Results}

To measure the most important physico-chemical parameters of detergents or mixtures of detergents, the CMC and the aggregation number, respectively, we developed a new assay that is based on a combination and improvement of existing protocols [8]-[10]. Due to the information gained with this assay, it is now possible to adjust buffer conditions of the purification and/or subsequent activity assay, in a controlled and almost highthroughput manner.

As recently described, fluorescence of Hoechst33342 in detergent solutions can be used to calculate CMCs for different detergent-water or detergent-buffer systems [1]. Hoechst33342 is highly fluorescence in hydrophobic environments but shows nearly no emission in water-based solutes. In addition to the method to measure the CMC of detergents [1], we further developed our method allowing now also the investigation of the influence of salts or $\mathrm{pH}$ on the CMCs of detergent mixtures as well as the determination of aggregation numbers.

To calculate aggregation numbers of detergents, pyrene quenching has been used extensively in the past [8][10]. Hoechst33342 absorption spectra and pyrene fluorescence emission spectra overlap. This implicates that Hoechst33342 can quench pyrene fluorescence in micelles as described in detail before [8] [9]. Figure 1 shows the fluorescence spectra of pyrene in the absence and presence of increasing Hoechst33342 concentrations. Here, the DDM concentration was 5 fold above CMC $(1 \mathrm{mM})$ and the pyrene concentration used was adjusted to an expected micelle to pyrene ratio of 10:1. Under these conditions no fluorescence peak at 470 nm was observed, indicating that no pyrene excimers were formed.

We first analyzed the partitioning of Hoechst33342 into different micelles. Partitioning coefficients (Pm) were calculated according to Equation (3) (see Materials and Methods). Hoechst33342 greatly migrates into the micelles as shown by the Pm values listed in Table 1. The Pm value for DDM is 2500 demonstrating, that almost all Hoechst33342 molecules are in the micellar phase under the conditions used in the assay. To phrase it differently, 2500 molecules of Hoechst33342 will be present in micelles, but only a single molecule of Hoechst33342 will reside in the aqueous phase. The same holds true for all other detergent tested (see Table 1).

Pyrene quenching by Hoechst33342 was analyzed measuring the emission peak of pyrene at $373 \mathrm{~nm}$ at different quencher concentrations. The intensity of pyrene fluorescence decreases with increasing quencher concentration (Figure 2). A plot of $\operatorname{lnF} / \mathrm{lnFo}$ versus quencher concentration results in a straight line from which the aggregation number can be calculated by using the slope. Here, an exactly measured CMC and as well as an accurate knowledge of the detergent concentration used in the assay are an absolute prerequisite (see Equation (4)).

Table 1. Calculated partitioning coefficients and literature values for CMC and aggregation number taken from www.affymetrix.com unless otherwise indicated.

\begin{tabular}{ccccc}
\hline Detergent & & Pm & $\boldsymbol{n}_{\text {agg }}$ Lit. & CMC Lit. (mM) \\
\hline DDM & Nonionic & 2500 & $78-149$ & 0.17 \\
DM & Nonionic & 390 & 69 & 1.8 \\
Triton X-100 & Nonionic & 3500 & $75-165$ & $0.15-0.24$ \\
FC-14 & Zwitterionic & - & 108 & 0.12 \\
LDAO & Zwitterionic & 1352 & $74^{\$}$ & $18-20$ \\
OG & Nonionic & 100 & $27-100$ & 8 \\
SDS & anionic & 80 & $62-101$ & 18 \\
\hline
\end{tabular}

\footnotetext{
§: [29], \$: [30].
} 


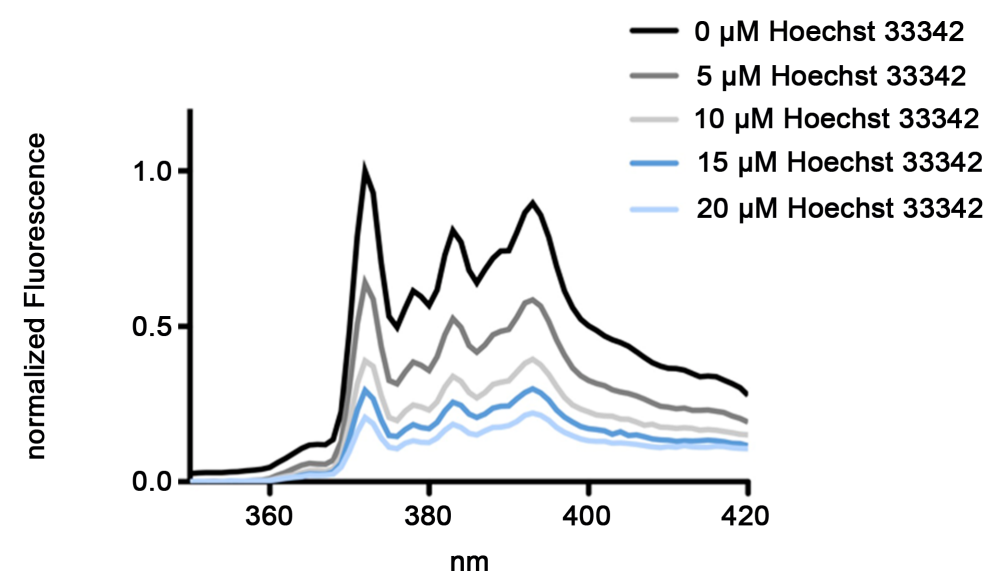

Figure 1. Corrected emission fluorescence spectra of a $5.0 \times 10^{-7} \mathrm{M}$ solution of pyrene in an aqueous solution of $1 \mathrm{mM}$ DDM at $25^{\circ} \mathrm{C}$. The black curve was collected in the absence of the quencher Hoechst33342; grey and blue curves were recorded in the presence of increasing (5 to $20 \mu \mathrm{M}$ ) quencher concentrations.



Figure 2. Quenching of Pyrene fluorescence by Hoechst33342 at three different detergent concentrations. The logarithm of the fluorescence intensity of the first vibronic peak of pyrene is shown as a function of Hoechst33342 concentration. Pyrene was excited at $335 \mathrm{~nm}$ and emission monitored at $372 \mathrm{~nm}$. Aggregation numbers were calculated from the slope of these three measurements and are 104, 135 and 154, respectively.

Pyrene quenching was measured for three different DDM concentrations. Increasing DDM concentrations resulted in a decrease in the slope of the logarithmic fluorescence ratio, due to the fact that the presence of an increased number of micelles decreases the probability to pair a donor and an acceptor molecule in the same micelle. The calculated aggregation numbers of 104, 135 and 154 show a similar tendency as measured by Lipfert et al. using SAXS (Table 2) [17]. To support our interpretation and the ease of our assay, we like to note that increasing aggregation numbers with increasing detergent concentrations were also observed for other detergents using different techniques for example SANS, NMR, and SAXS as well (Table 2) [8] [17]-[19], but in our case they are determined using a standard equipment present in virtually any biochemically focused laboratory.

Furthermore, we analyzed the influence of salt on the behavior of the detergent in terms of CMC and aggregation number. Information about the influence of salt, $\mathrm{pH}$, denatured reagents or further parameters on the physico-chemical nature of a detergent micelle can be very useful to optimize buffer conditions especially to reduce the amount of free detergent. Therefore, we analyzed the influence of increasing ionic strength on the aggregation number. The non-ionic detergent DDM shows only small changes in aggregation number under increasing 
Table 2. Concentration dependency of aggregation numbers.

\begin{tabular}{|c|c|c|c|c|}
\hline Detergent & Concentration [mM] & Aggregation number & Method & Literature \\
\hline DDM & $\begin{array}{l}1 \\
2 \\
3\end{array}$ & $\begin{array}{l}104 \\
135 \\
154\end{array}$ & $\begin{array}{l}\text { Steady state fluorescence } \\
\text { quenching }\end{array}$ & This study \\
\hline DDM & $\begin{array}{c}5 \\
10\end{array}$ & $\begin{array}{l}136 \\
140\end{array}$ & SAXS & [17] \\
\hline SDS & $\begin{array}{c}100 \\
200 \\
300 \\
400 \\
500 \\
30 \\
70 \\
100 \\
125 \\
160 \\
250\end{array}$ & $\begin{array}{c}69 \\
78 \\
85 \\
90 \\
95 \\
84 \\
100 \\
113 \\
122 \\
129 \\
142\end{array}$ & NMR & [19] \\
\hline Triton X-100 & $\begin{array}{l}1.6 \\
3.2 \\
4.8 \\
6.4\end{array}$ & $\begin{array}{l}111 \\
125 \\
140 \\
156\end{array}$ & $\begin{array}{c}\text { Steady state fluorescence } \\
\text { quenching }\end{array}$ & [8] \\
\hline OG & $\begin{array}{c}50 \\
75 \\
100 \\
150\end{array}$ & $\begin{array}{c}83 \\
99 \\
111 \\
120\end{array}$ & SAXS & [17] \\
\hline
\end{tabular}

salt concentrations (Figure 3(a)) [20]. Through addition of $800 \mathrm{mM} \mathrm{NaCl}$, the aggregation number of DDM increases only little from 98 to 105 (+7\%). We exemplarily show the effect of the $\mathrm{NaCl}$ concentration on the aggregation number of SDS, an ionic detergent. Here, a more drastic change could be detected. The addition of already $250 \mathrm{mM} \mathrm{NaCl}$ resulted in a change of almost 25\% when compared to SDS in buffer lacking NaCl (Figure 3(b)). These data are line with data obtained by ultracentrifugation [21], time-resolved fluorescence quenching [22], light scattering [23] or EPR [24].

Often large micelles might stabilize an IMP, but might interfere with activity or for example with the formation of crystal contacts on the other hand [7]. With the pyrene quenching method, it is possible to measure several detergent mixtures in a 96-well plate reader within a couple of hours. DDM is a very widely used detergent for IMP purification and activity measurements. Therefore, we decide to mix DDM with other detergents in the studies described below and shown in Figure 4. Different ratios of DDM and a second detergent where analyzed for their influence on CMC and aggregation number and plotted against the molar ratio of added detergent. Most of the calculated CMCs showed an almost ideal mixing behavior according to Equation (7), shown in Figure 4(b), Figure 4(c) and Figure 4(e) (straight line). Here, we refer to the thermodynamic model described by Clint and Motomura [15] [25]. In our study, however, little deviation from ideal behavior is observed. One example for non-ideal behavior of a detergent mixture is DDM mixed with SDS as depicted in Figure 4(f). Here, the values of CMC of a 1:4 and 1:9 mixture of DDM and SDS lie below the expected values demonstrating that the influence of SDS on the CMC of DDM is rather low. This behavior was also measured by Zhang et al. using surface tension measurements [26] [27]. The opposite is true for Triton $\times 100$. In Figure 4(d), mixing of DDM and Triton is shown. The measured values for CMC are above the theoretical values when the ratio of Triton $\times$ 100 to DDM is higher than 0.5 (black triangles). This non-ideal behavior could also be detected for the aggregation numbers of these mixtures. A drastic decrease of the aggregation number for DDM/SDS mixtures could only be measured after $80 \%$ of all detergent present in the sample was SDS, again showing the small impact of SDS on DDM micelle formation (black rectangles in Figure 4(f)). A much higher influence again shows Triton $\times 100$ (Figure 4(d)). For a 20\% Triton $\times 100$ in a DDM solution, the aggregation number measured is even beyond that of the two solutions of the pure components. Thus, small amounts of Triton $\times 100$ really forces DDM micelles to swell. Increasing the amount of Triton $\times 100$ in the solution further reduces the aggregation 


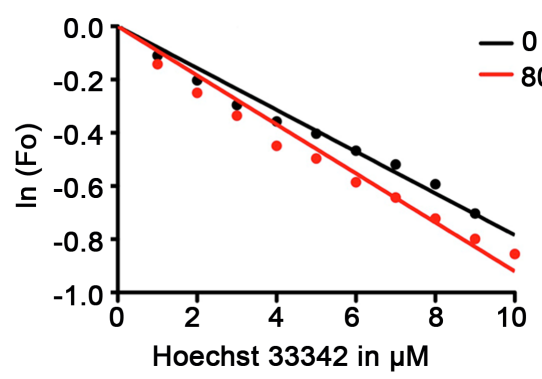

(a)

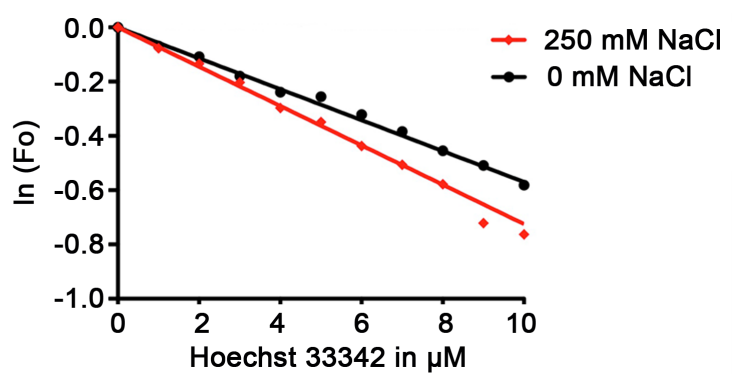

(b)

Figure 3. Salt dependency of aggregation number of SDS. Increasing salt concentrations result in a swelling of the micelle. Aggregation numbers calculated from this plots resulted in a value of 68 and 86 monomers per micelle, respectively.

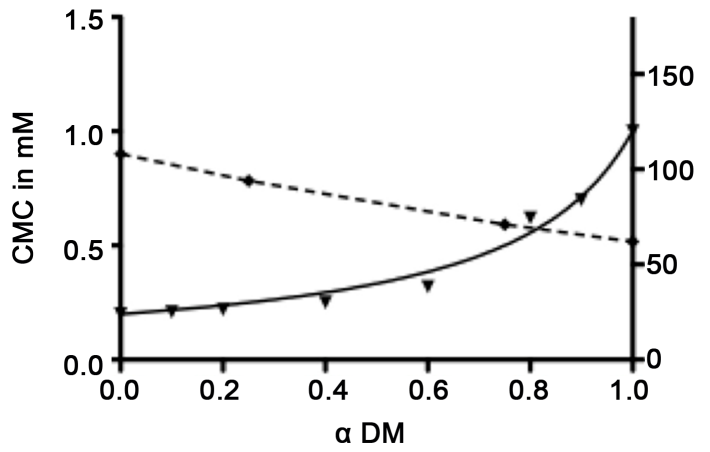

(a)

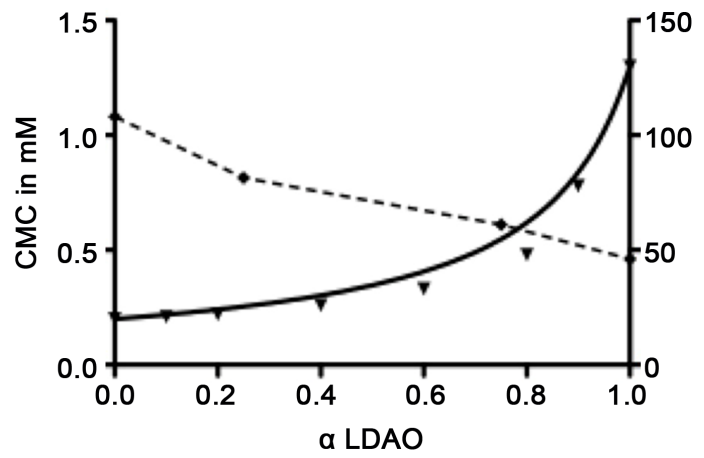

(c)

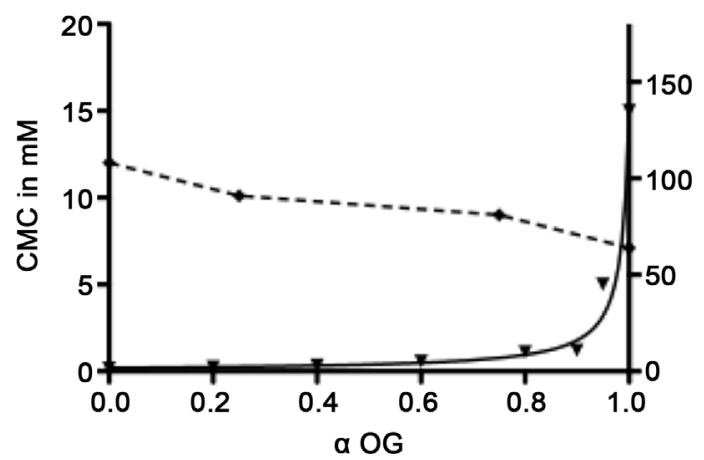

(e)

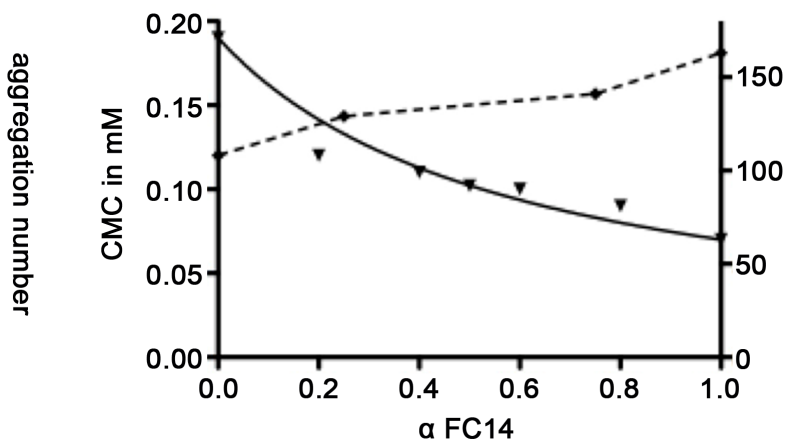

(b)

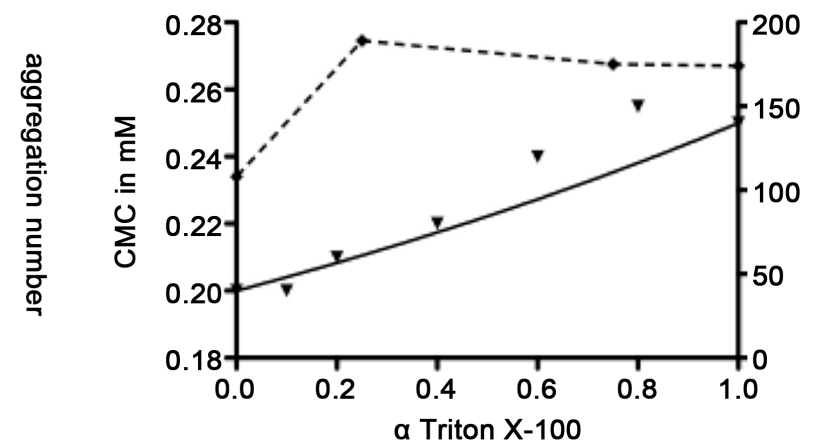

(d)

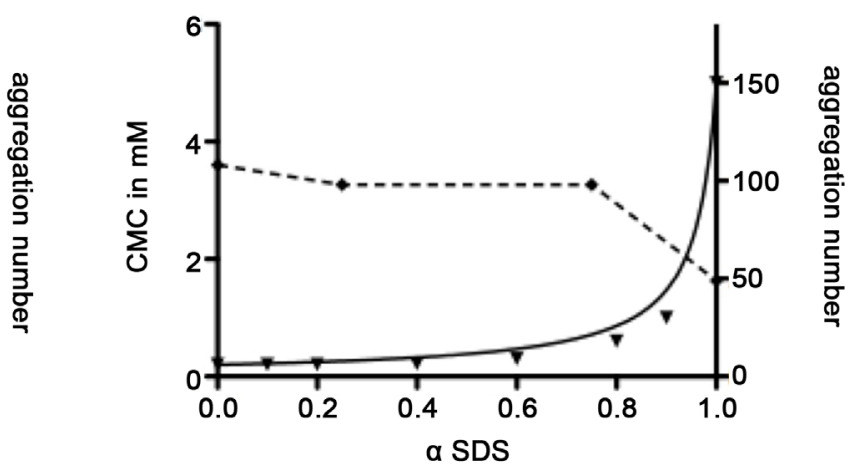

(f)

Figure 4. CMC and aggregation number determination of mixtures of DDM with different detergents via the 96-well plate reader set-up. Triangles show the behavior of the CMC at different ratios of a second detergent. Quadrangles indicate aggregation number measured for different detergent mixtures. 
number but never drops below that of the aggregation number of a $100 \%$ Triton $\times 100$ solution. This demonstrates that although theoretically it is possible to calculate the behavior of detergent mixture while mixing, the two detergents do not behave ideally.

These findings strongly recommend investigating the CMC and aggregation number of the detergent mixture in a buffer of interest should be investigated before the experiment is started. Our method allows this point to be addressed in a matter of a couple of hours, which could help finding an optimal buffer system for the planned experiment.

\section{Discussion}

Quenching of pyrene fluorescence by Hoechst33342 can be used as a simple and rapid method to determine aggregation numbers of single detergents and detergent mixtures. Together with the usage of a 96well plate reader, ten or more detergent mixtures can be measured in less than one hour. Although other fluorophor combinations can be used, pyrene was chosen due to its long fluorescence lifetime ( $\approx 400 \mathrm{ns)}$ [28] and similar to Hoechst33342 it is relatively cheap and widely available.

Hoechst33342 greatly partitions into the micellar phase and shows an overlap with the fluorescence spectrum of pyrene. Furthermore, it does almost not fluorescence in the region of the pyrene fluorescence peak maximum (273 nm). Hoechst33342 fluorescence can be used to determine CMC values [1]. Combining these two characteristics provides the opportunity to determine the aggregation number even in a 96-well plate as shown in this study. Furthermore, the physical behavior of detergent mixtures can be measured using basically every imaginable ratio of two or more different detergents. Here, we used DDM as detergent to demonstrate the efficiency of our set-up. To the best of our knowledge, this method is applicable to any detergent/mixture.

We show that not all detergents mix very well with DDM (e.g. SDS or Triton X-100). A comparison of our data with theoretically calculated mixing behaviors shows deviances of more than $50 \%$. The reason for this behavior remains unknown. Therefore, one has to be even more careful when mixing detergents with known large differences in their CMC or head groups (for example nonionic or ionic).

Another argument for determining these parameters for every buffer individually is the wide range of known CMCs and aggregation numbers. Anatrace (www.anatrace.com) states that the CMC values of OG lie between 18 to $20 \mathrm{mM}$ depending on the buffer system used. Even more drastic is the variance in aggregation number, which can be between 27 and 100 monomers per micelle according to Anatrace. Another example is CMC of LDAO indicated as being between 1 and $2 \mathrm{mM}$, a variance of 100\%. Here, our set-up provides an easy and robust approach to determine appropriately the cmc and aggregation number under the conditions of the exact experimental conditions.

\section{Acknowledgements}

We thank Thorsten Jumpertz, and André Abts for valuable discussions. This work was funded by the consortiaEDICT (European Initiative on Channels and Transporters) FP7 Theme [Health-2007-2.1.1-5] to L.S.

\section{References}

[1] Jumpertz, T., Tschapek, B., Infed, N., Smits, S.H., Ernst, R. and Schmitt, L. (2011) High-Throughput Evaluation of the Critical Micelle Concentration of Detergents. Analytical Biochemistry, 408, 64-70. http://dx.doi.org/10.1016/j.ab.2010.09.011

[2] Paradies, H.H. (1980) Shape and Size of a Nonionic Surfactant Micelle. Triton X-100 in Aqueous Solution. The Journal of Physical Chemistry, 84, 599-607. http://dx.doi.org/10.1021/j100443a008

[3] Lund, S., Orlowski, S., de Foresta, B., Champeil, P., le Maire, M. and Moller, J.V. (1989) Detergent Structure and Associated Lipid as Determinants in the Stabilization of Solubilized $\mathrm{Ca}^{2+}$-ATPase from Sarcoplasmic Reticulum. The Journal of Biological Chemistry, 264, 4907-4915.

[4] Infed, N., Hanekop, N., Driessen, A.J., Smits, S.H. and Schmitt, L. (2011) Influence of Detergents on the Activity of the ABC Transporter LmrA. Biochimica et Biophysica Acta, 1808, 2313-2321. http://dx.doi.org/10.1016/j.bbamem.2011.05.016

[5] Screpanti, E., Padan, E., Rimon, A., Michel, H. and Hunte, C. (2006) Crucial Steps in the Structure Determination of the $\mathrm{Na}^{+} / \mathrm{H}^{+}$Antiporter NhaA in Its Native Conformation. Journal of Molecular Biology, 362, 192-202. http://dx.doi.org/10.1016/j.jmb.2006.07.019 
[6] Lemieux, M.J., Song, J., Kim, M.J., Huang, Y., Villa, A., Auer, M., Li, X. D. and Wang, D.N. (2003) Three-Dimensional Crystallization of the Escherichia coli Glycerol-3-Phosphate Transporter: A Member of the Major Facilitator Superfamily. Protein Science, 12, 2748-2756. http://dx.doi.org/10.1110/ps.03276603

[7] Michel, H. (1983) Crystallization of Membrane Proteins. Trends in Biochemical Sciences, 8, 56-59. http://dx.doi.org/10.1016/0968-0004(83)90390-0

[8] Tummino, P.J. and Gafni, A. (1993) Determination of the Aggregation Number of Detergent Micelles Using SteadyState Fluorescence Quenching. Biophysical Journal, 64, 1580-1587. http://dx.doi.org/10.1016/S0006-3495(93)81528-5

[9] Turro, N.J. and Yekta, A. (1978) Luminescent Probes for Detergent Solutions. A simple Procedure for Determination of Mean Aggregation Number of Micelles. Journal of the American Chemical Society, 100, 5951-5952. http://dx.doi.org/10.1021/ja00486a062

[10] Infelta, P.P. (1978) Fluorescence Quenching in Micellar Solutions and Its Application to the Determination of Aggregation Numbers. Chemical Physics Letters, 61, 88-91. http://dx.doi.org/10.1016/0009-2614(79)85092-7

[11] Prive, G.G. (2007) Detergents for the Stabilization and Crystallization of Membrane Proteins. Methods, 41, $388-397$. http://dx.doi.org/10.1016/j.ymeth.2007.01.007

[12] Pastor, O., Junquera, E. and Aicart, E. (1998) Hydration and Micellization Processes of $n$-Octyl- $\beta$-D-Glucopyranoside in Aqueous Solution. A Thermodynamic and Fluorimetric Study in the Absence and Presence of Salts. Langmuir, 14, 2950-5957. http://dx.doi.org/10.1021/la9708445

[13] Atik, S.S. and Singer, L.A. (1978) Nitroxyl Radical Quenching of the Pyrene Fluorescence in Micellar Environments. Development of a Kinetic Model for Steady-State and Transient Experiments. Chemical Physics Letters, 59, 519-524. http://dx.doi.org/10.1016/0009-2614(78)85032-5

[14] Hansson, P., Jönsson, B., Ström, C. and Södermann, O. (2000) Determination of Micellar Aggregation Numbers in Dilute Surfactant Systems with the Fluorescence Quenching Method. The Journal of Physical Chemistry B, 104, 34963506. http://dx.doi.org/10.1021/jp992444r

[15] Clint, J.H. (1974) Micellization of Mixed Nonionic Surface Active Agents. Journal of the Chemical Society, Faraday Transactions, 71, 1327-1334. http://dx.doi.org/10.1039/f19757101327

[16] Sarmoria, C., Puvvada, S. and Blankschtein, D. (1992) Prediction of Critical Micelle Concentrations of Nonideal Binary Surfactant Mixtures. Langmuir, 8, 2690-2697. http://dx.doi.org/10.1021/la00047a019

[17] Lipfert, J., Columbus, L., Chu, V.B., Lesley, S.A. and Doniach, S. (2007) Size and Shape of Detergent Micelles Determined by Small-Angle X-Ray Scattering. The Journal of Physical Chemistry B, 111, 12427-12438. http://dx.doi.org/10.1021/jp073016l

[18] Bezzobotnov, V.Y., Borbély, S., Cser, L., Faragó, B., Gladkih, I.A., Ostanevich, Y.M. and Vass, S. (1988) Temperature and Concentration Dependence of Properties of Sodium Dodecyl Sulfate Micelles Determined from Small Angle Neutron Scattering Experiments. The Journal of Physical Chemistry, 92, 5738-5743. http://dx.doi.org/10.1021/j100331a038

[19] Gangabadage, C.S., Najda, A., Bogdan, D., Wijmenga, S.S. and Tessari, M. (2008) Dependence of the Size of a Protein-SDS Complex on Detergent and $\mathrm{Na}^{+}$Concentrations. The Journal of Physical Chemistry B, 112, 4242-4245. http://dx.doi.org/10.1021/jp710045e

[20] Bucci, S., Fagotti, C., Degiorgio, V. and Piazza, R. (1991) Small-Angle Neutron-Scattering Study of Ionic-Nonionic Mixed Micelles. Langmuir, 7, 824-826. http://dx.doi.org/10.1021/la00053a002

[21] Doughty, D.A. (1979) Isopiestic Compositions of Aqueous Ionic Surfactant Systems as a Measure of Preferential Interactions. Application to the Determination of Micelle Aggregation Numbers by Equilibrium Ultracentrifugation. The Journal of Physical Chemistry, 83, 2621-2628. http://dx.doi.org/10.1021/j100483a014

[22] Lianos, P. and Zana, R. (1980) Use of Pyrene Excimer Formation to Study the Effect of Sodium Chloride on the Structure of Sodium Dodecyl Sulfate Micelles. The Journal of Physical Chemistry, 84, 3339-3341. http://dx.doi.org/10.1021/j100462a003

[23] Emerson, M.F. and Holtzer, A. (1967) On the Ionic Strength Dependence of Micelle Number II. The Journal of Physical Chemistry, 71, 1898-1907. http://dx.doi.org/10.1021/j100865a057

[24] Bales, B.L., Messina, L., Vidal, A. and Peric, M. (1998) Precision Relative Aggregation Number Determinations of SDS Micelles Using a Spin Probe. A Model of Micelle Surface Hydration. The Journal of Physical Chemistry B, 102, 10347-10358. http://dx.doi.org/10.1021/jp983364a

[25] Motomura, K., Yamanaka, M. and Aranato, M. (1984) Thermodynamic Consideration of the Mixed Micelle of Surfactants. Colloid \& Polymer Science, 262, 948-955. http://dx.doi.org/10.1007/BF01490027

[26] Zhang, R., Zhang, L. and Somasundaran, P. (2003) Study of Mixtures of $n$-Dodecyl- $\beta$-D-Maltoside with Anionic, Cationic, and Nonionic Surfactant in Aqueous Solutions Using Surface Tension and Fluorescence Techniques. Journal of 
Colloid and Interface Science, 278, 453-460. http://dx.doi.org/10.1016/j.jcis.2004.06.045

[27] Columbus, L., Lipfert, J., Jambunathan, K., Fox, D.A., Sim, A.Y., Doniach, S. and Lesley, S.A. (2009) Mixing and Matching Detergents for Membrane Protein NMR Structure Determination. Journal of the American Chemical Society, 131, 7320-7326. http://dx.doi.org/10.1021/ja808776j

[28] Turro, N.J., Graetzle, M. and Braun, A.M. (1980) Photophysical and Photochemical Processes in Micellar Systems. Angewandte Chemie International Edition in English, 19, 675-696. http://dx.doi.org/10.1002/anie.198006751

[29] Herrmann, K.W. (1962) Non-Ionic-Cationic Micellar Properties of Dimethyldodecylamine Oxide. The Journal of Physical Chemistry, 66, 295-300. http://dx.doi.org/10.1021/j100808a025

[30] Herrmann, K.W. (1966) Micellar Properties of Some Zwitterionic Surfactants. Journal of Colloid and Interface Science, 22, 352-359. http://dx.doi.org/10.1016/0021-9797(66)90015-4 